\title{
Surgical management of sternoclavicular joint infections
}

\author{
Harold M. Burkhart, MD \\ Claude Deschamps, MD \\ Mark S. Allen, MD \\ Francis C. Nichols III, MD \\ Daniel L. Miller, MD \\ Peter C. Pairolero, MD
}

\footnotetext{
From the Division of General Thoracic Surgery, Mayo Clinic and Mayo Foundation, Rochester, Minn.

Read at the Eighty-first Annual Meeting of The American Association for Thoracic Surgery, San Diego, Calif, May 6-9, 2001.

Received for publication May 30, 2002; revisions requested July 11, 2002; revisions received Sept 8, 2002; accepted for publication Sept 17, 2002.

Address for reprints: Claude Deschamps, MD, Division of General Thoracic Surgery, Mayo Clinic and Mayo Foundation, 200 First St SW, Rochester, MN 55905 (E-mail: deschamps.claude@mayo.edu).

J Thorac Cardiovasc Surg 2003;125:945-9

Copyright (C) 2003 by The American Association for Thoracic Surgery

0022-5223/2003\$30.00+0

doi:10.1067/mtc.2003.172
}

Objective: Sternoclavicular joint infections are rare, and their management is controversial. We reviewed our experience with the surgical management of this condition.

Methods: From August 1988 to August 2001, 26 patients (16 men and 10 women) were treated surgically for infected sternoclavicular joints. The median age was 56 years (range, 20-77 years). Patients who had a recent previous median sternotomy were excluded.

Results: All patients were symptomatic. Pain was present in 21 patients, swelling in 14 patients, fever in 11 patients, and erythema in 9 patients. Associated conditions included recent or ongoing infections in other areas in 12 patients (pneumonia in 4 patients, multiple joint infections in 2 patients, and other in 6 patients) and an indwelling central venous catheter in 1 patient. Five patients had a history of trauma in the region of the joint. Four patients had prior joint incision and drainage. Unilateral sternoclavicular joint resection was done in 18 patients, bilateral resection in 2 patients, and incision and drainage with debridement in 6 patients. Wound culture results were positive in 24 patients, and the most common organism isolated was Staphylococcus aureus $(\mathrm{n}=17)$. Eleven patients had transposition of the ipsilateral pectoralis major muscle to obliterate residual space and to reconstruct the chest wall. Two (7.7\%) patients had complications, and 1 died (operative mortality, $3.8 \%$ ). Follow-up was complete in all 25 operative survivors and ranged from 2 months to 10 years (median, 25 months). Twenty-one patients are alive without symptoms, infection, or limitations in range of motion. Four patients have died as a result of causes unrelated to their joint infections.

Conclusions: Symptomatic sternoclavicular joint infections often require surgical intervention. Surgical resection combined with muscle transposition provides effective long-term outcome.

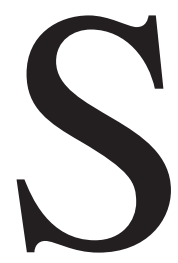

ternoclavicular joint (SCJ) infections are rare. Their cause and presentation is not well characterized. Similarly, the management of SCJ infections is controversial. ${ }^{1}$ Management strategies have ranged from conservative antibiotic therapy to surgical resection of the SCJ with or without muscle transposition. The purpose of this review is to analyze our experience with the surgical management of SCJ infections.

\section{Patients and Methods}

Between August 1, 1988, and August 1, 2001, all patients who underwent surgical management of SCJ infection at the Mayo Clinic in Rochester, Minn, were reviewed. Patients who had SCJ infection as a result of an infected median sternotomy were excluded. The records of these patients were analyzed for age, sex, preoperative symptoms, roentgenographic findings, operative procedures, microbiology data, and clinical outcome. Operative mortality included those patients who died within the first 30 days after the operation and those who 
died later but during the same hospitalization. Follow-up was obtained through outpatient clinic visits, correspondence with local physicians, and telephone interviews.

There were 16 men and 10 women in the study. The median age was 56 years and ranged from 20 to 77 years. Twelve patients had recent or ongoing infections in other locations that included pneumonia in 4 patients, sepsis with multiple joint infections in 2 patients, and spontaneous bacterial peritonitis, abdominal abscess, infected knee prosthesis, cutaneous ulcer infection, hand cellulitis, and bacteremia in 1 patient each. Five patients had a history of trauma in the region of the joint, and one had an ipsilateral indwelling subclavian venous catheter. Other comorbidities included diabetes mellitus in 8 patients, connective tissue disorders in 3 patients, steroid use in 3 patients, radiation therapy after a modified radical mastectomy in 2 patients, and acute myelocytic leukemia in 1 patient. Presenting signs and symptoms were present in all 26 patients and included pain in $21(81.7 \%)$ patients, swelling in $14(53.8 \%)$ patients, fever in $11(42.3)$ patients, erythema in $9(34.6 \%)$ patients, and purulent drainage in $3(11.5 \%)$ patients. The SCJ infection was on the right in $16(61.5 \%)$ patients, on the left in $8(30.7 \%)$ patients, and bilateral in $2(7.7 \%)$ patients. Parenteral antibiotics were administered preoperatively in 12 (46.1\%) patients and ranged from 2 to 48 days, with a median of 14 days before surgical intervention.

A chest roentgenograph was performed in all patients and demonstrated a mass in the region of the SCJ in 4 patients and bone destruction in 2 patients. The remaining 20 (81\%) chest roentgenographs were interpreted as normal. Computed tomography (CT) of the chest was performed in 22 patients and demonstrated SCJ swelling in 10 patients, bone destruction in 9 patients, an abscess in 9 patients, and a mass in 2 patients. A radionuclide bone scan was performed in 8 patients, and the results were abnormal in 7 patients.

Wound cultures were obtained in all 26 patients, and results were positive in $24(92.3 \%)$ patients. Staphylococcus aureus was isolated in 17 patients (65.4\%), Streptococcus species in 4 (15.4\%) patients, Escherichia coli in $2(7.7 \%)$ patients, and multiple organisms in $1(3.8 \%)$ patient. Blood cultures were obtained in 19 $(73.0 \%)$ patients and demonstrated the same organism as found in the wound in 11 patients.

SCJ resection was performed in 20 patients (unilateral in 18 patients and bilateral in 2 patients), and incision and drainage with debridement was performed in 6 patients. In performing SCJ resections, our preference has been to make a supraclavicular incision that extends from the medial third of the involved clavicle toward the suprasternal notch and then down the midline to the sternomanubrial junction. The attachment of the sternocleidomastoid muscle is separated from the clavicle, and the pectoralis major muscle is dissected from the manubrium and anterior chest wall for several centimeters toward the midaxillary line. The clavicle is then divided just lateral to the SCJ, followed by midline division of the manubrium to just below the first costochondral arch. The entire first costochondral arch is now removed, and the SCJ is resected en bloc, with care taken to avoid damage to the underlying subclavian vein and internal thoracic vessels.

In the patients who had SCJ resection, primary wound closure was done in 2 patients, and delayed closure was done in 12 patients. In the remaining 6 patients, the wound was closed by means of secondary intention. Four of the 20 patients who had SCJ resection had prior incision and drainage. In the 6 patients who had SCJ incision and drainage with debridement, the wounds in 2 patients were closed primarily, 2 patients had delayed closure, and 2 patients healed by secondary intention. Eleven $(55 \%)$ of the 20 patients undergoing SCJ resection underwent transposition of the ipsilateral pectoralis major muscle to obliterate residual space and reconstruct the chest wall. Twenty $(77.0 \%)$ patients received postoperative antibiotics for a median of 42 days (range, 14-56 days).

\section{Results}

Median hospitalization was 14 days (range, 3-110 days). There was one operative death (mortality, 3.8\%). This patient had multiple joint infections and underwent bilateral SCJ resections. She died 3 months after resection but during the same hospitalization as a result of sepsis and multisystem organ failure. At the time of death, the area of joint resection had healed and had no evidence of drainage. Two (7.7\%) patients had postoperative complications. One patient with SCJ resection who had delayed closure without muscle transposition had recurrent infection 9 days after closure and required reopening. This wound eventually healed by secondary intention. The other complication occurred in a patient who had unstable angina and required coronary artery bypass grafting and mitral valve repair in the early postoperative period.

Follow-up was complete in all 25 operative survivors and ranged from 2 months to 10 years (median, 26 months). Twenty-one patients are currently alive without symptoms, infection, or limitations in range of motion. Four patients have died as a result of causes unrelated to their joint infections. Causes of death included acute myelocytic leukemia, cerebral vascular accident, hepatic failure, and accidental hypothermia.

\section{Discussion}

The SCJ is synovial lined and composed of the inferior, medial clavicular head, the superior lateral notch of the manubrium, and the cartilage of the first rib. Clinical infections of this joint are rare but, when present, result in abscess formation in $20 \%$ of patients. ${ }^{2}$ Because the joint capsule is unable to distend, infection quickly spreads beyond the joint, leading to fistula formation, cutaneous abscesses, or, rarely, mediastinitis. ${ }^{3-4}$ Given the joint's close relationship to the mediastinum, pleural cavity, and brachiocephalic structures, thoracic surgeons will often be consulted and should be familiar with the surgical management of an infected SCJ.

SCJ infection has been reported in association with central venous catheters, intravenous drug abuse, multijoint infections, local trauma, and immunosuppression from diabetes mellitus, chronic steroid use, hemodialysis, and HIV infection.1,5-9 SCJ infections have also been reported to occur spontaneously in otherwise healthy patients. ${ }^{10}$ Carlos 


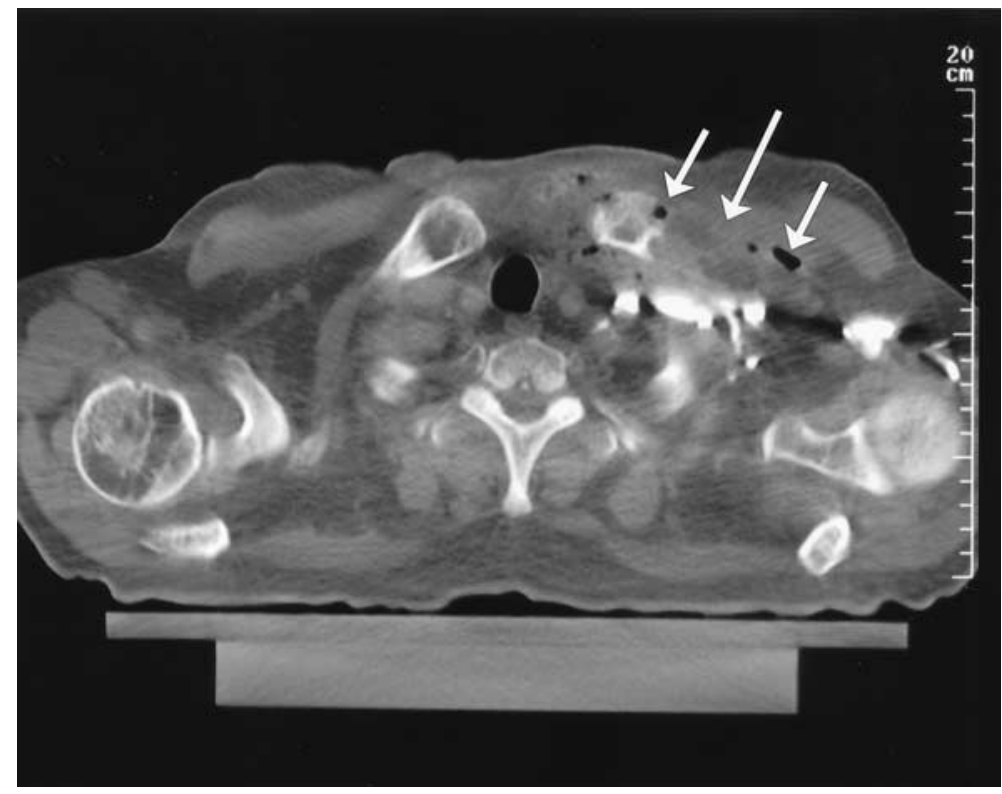

Figure 1. CT at the level of the left SCJ demonstrating bone destruction with air (arrows) in the surrounding soft tissues.

TABLE 1. SCJ infections: Reported series

\begin{tabular}{|c|c|c|c|c|c|}
\hline Author & $\begin{array}{l}\text { Patients } \\
\text { (n) }\end{array}$ & $\begin{array}{l}\text { Contributing factors } \\
\text { (n) }\end{array}$ & $\begin{array}{l}\text { Treatment } \\
\text { (n) }\end{array}$ & $\begin{array}{l}\text { Muscle } \\
\text { flap (n) }\end{array}$ & Outcome \\
\hline Carlos and associates 5 & 8 & Central venous catheter (5) & $\begin{array}{l}\text { Resection (4); } \\
\quad \text { I \& D (4) }\end{array}$ & $\begin{array}{l}4 \\
0\end{array}$ & Good \\
\hline Song and associates ${ }^{1}$ & 7 & Immunosupression (4) & $\begin{array}{l}\text { Resection (6); } \\
\quad \text { I \& D (1) }\end{array}$ & $\begin{array}{l}6 \\
0\end{array}$ & Good \\
\hline Current study & 26 & $\begin{array}{l}\text { Immunosupression (4); } \\
\text { other infections (12) }\end{array}$ & $\begin{array}{l}\text { Resection (20); } \\
\quad \text { I \& D (6) }\end{array}$ & $\begin{array}{l}11 \\
0\end{array}$ & Good \\
\hline
\end{tabular}

I\& $D$, Incision and drainage.

and colleagues 5 reported on a surgical series of 8 patients, with the suspected source of infection being a subclavian vein catheter in 4 patients and a subclavian vein pacemaker in 1 patient. In the series reported by Song and associates, ${ }^{1}$ the most common risk factor for SCJ infection was immunosuppression. Similarly, a variety of underlying causes was found in our patients.

Patients with suspected SCJ infection should be evaluated with both chest roentgenography and chest CT. Although chest roentgenography in our experience has a low likelihood of diagnosing SCJ infection, it might be useful in evaluating other types of thoracic problems. Although our preference has been to image the SCJ with CT (Figure 1), magnetic resonance scans can be of equal value. If the imaging studies are equivocal, however, a radionuclide bone scan might be helpful. If no evidence of abscess or bone destruction is found and an SCJ infection is suspected, parenteral antibiotics should be instituted. When doubt persists as to the diagnosis, incision and drainage can be performed to obtain tissue sample for histologic examination and culture. However, when either abscess or bone destruction exists, we advocate resection of the SCJ.

Song and associates ${ }^{1}$ reported that 5 of their 6 patients undergoing SCJ resection had previous drainage and debridement. More than two thirds of our patients required SCJ resection, and $15 \%$ had undergone previous incision and drainage. Our results and those from other reported series $^{1,5}$ support aggressive surgical management (Table 1). We have previously reported the utility of muscle transposition in controlling local infection in other anatomic loca- 
tions. ${ }^{11}$ For lesions of the anterior chest wall, transposition of the pectoralis major muscle on the basis of the thoracoacromial neurovascular leash without division of the humeral attachments is ideal. This provides for excellent local infection control and obliteration of residual space, as well as maintaining pectoralis major function.

In conclusion, SCJ infections often require surgical intervention. Joint resection combined with muscle transposition is effective and results in both a healed chest wall without evidence of infection and preservation of shoulder function in most patients.

\section{References}

1. Song HK, Guy TS, Kaiser LR, Shrager JB. Current presentation and optimal surgical management of sternoclavicular joint infections. Ann Thorac Surg. 2002;73:427-31.

2. Wohlgethan JR, Newberg AH, Reed JI. The risk of abscess from sternoclavicular arthritis. J Rheumatol. 1988;15:1302-6.

3. Asnis DS, Dhaliwal GS. Bilateral sternoclavicular joint septic arthritis presenting as cutaneous abscesses. Clin Infect Dis. 1994;19:964-6.

4. Koroscil TM, Valen PA. Sternoclavicular septic arthritis due to Haemophilis influenzae. South Med J. 1990;83:1469-71.

5. Carlos GN, Kesler KA, Coleman JJ, Broderick L, Turrentine MW, Brown JW. Aggressive surgical management of sternoclavicular joint infections. J Thorac Cardiovasc Surg. 1997;113:242-7.

6. Renoult E, Lataste A, Jonon B, Testevuide, Kessler M. Sternoclavicular joint infection in hemodialysis patients. Nephron. 1990;56:212-3.

7. Covelli M, Lapadula G, Pipitone N, Numo R, Pipitone V. Isolated sternoclavicular joint arthritis in heroin addicts and/or HIV positive patients: three cases. Clin Rheumatol. 1993;12:422-5.

8. Nair V. Sternoclavicular arthritis: an unusual complication of drug abuse. J Med Soc NJ. 1975;72:519-20.

9. Yood YA, Goldenberg DL. Sternoclavicular joint arthritis. Arthritis Rheum. 1980;23:232-9.

10. Gillis S, Friedman B, Caraco Y, Blankstein A, Yellin A, Friedman G. Septic arthritis of the sternoclavicular joint in healthy adults. J Intern Med. 1990;228:275-8.

11. Pairolero PC, Arnold PG, Harris JB. Long-term results of pectoralis major muscle transposition for infected sternotomy wounds. Ann Surg. 1991;213:583-9.

\section{Discussion}

Dr Malcolm M. DeCamp, Jr (Cleveland, Ohio). SCJ infections are uncommon, but with an increasing number of immunocompromised hosts in our practice, as well as patients with indwelling intravascular prostheses, it is perhaps an entity that is on the rise. Dr Burkhart and his colleagues from the Mayo Clinic are to be congratulated for their complete report of this unusual but challenging problem. They build on their published experience with the use of muscle flaps to help remedy a variety of thoracic defects and infections. This current work is arguably the largest surgical series of SCJs managed to date. As leaders in the field of thoracic muscle flaps, the authors can use their experience to help guide us.

I have several questions. Is there any way to define the denominator in terms of SCJ infection? I suspect that is difficult, and therefore, if not, can the authors provide criteria to help the surgeons decide when to incise and drain and when to resect? Were there clinical factors that predicted success with either intervention?

Eleven of your 20 resected joint spaces were reconstructed with ipsilateral pectoral muscle. Were there clinical factors favoring immediate muscle flap closure versus delayed closure versus those that were allowed to heal by secondary intention?

How is postoperative shoulder function assessed? Did the degree of clavicular resection affect shoulder function or chronic pain?

Finally, can you comment on the unusual problem of bilateral SCJ resections? Unfortunately, one of your patients died. Nevertheless, how were these wounds managed? Was the entire manubrium resected? What was the long-term functional result in your one survivor?

Dr Burkhart. Thank you for your comments, Dr DeCamp. You point out a couple of the limitations in this report, and the first one is whether we can define the common denominator. No matter what we tried to evaluate, looking at all the patients in the medical wards and surgical wards who had been admitted to the hospital over that time period, there were only 2 or 3 that came up as actually having SCJ infections that were not treated surgically, and we do not know if there are more. However, in predicting when to do incision and drainage and when to do a resection, I think we have done resections on the last several patients, and I think that is our preferred route in almost all patients. The patient undergoing incision and drainage would typically be one who could not undergo an operation for medical reasons, sepsis, or hemodynamic instability. Possibly, we might be unsure about the diagnosis; the patient might have erythema on the joint and we would want to see whether an infection was in fact present. However, if any of the patients specifically have an abscess or bone destruction or ongoing sepsis with that as a point of infection, have a recurrence of the infection after incision and drainage, or, in addition, have persistence of infection despite adequate medical therapy for 2 weeks or so, we try to perform a resection. As far as when to use flap closure, both immediate and delayed, only about $55 \%$ of the resections had muscle flaps. I will first start by saying that we do get plastic surgery involved in the management of the wound with all of these resections, at least at the time of the operation. When deciding on whether to do a flap, the biggest question is whether the patient has adequate soft tissue to cover the operative site. If patients have a lot of soft tissue in the upper chest and, after the resection is done, there is just a small defect and they have quite a bit of subcutaneous tissue and muscle, then the wound is closed by secondary intention. However, if they are thin, there is a large defect, or their brachiocephalic structures are exposed, we tend to do a muscle flap.

As you pointed out, primary closure was used in 2 patients. In most of them, because of the nature of the infection, the wound is packed for a few days before being closed primarily. I think it is a rarity that we close such wounds primarily. Of those 2 patients, 1 of them had an abscess on CT scan and 1 of them had bone destruction on a chest radiograph; neither of them had any comorbidity factors or diabetes, which would have made us think that they would have a difficult time healing. Therefore, in those 2 patients the wound was actually closed immediately and they did well.

Postoperative functional status was the other limitation of this study. We do not have any objective data. This was all purely subjective, either seeing the patients in the clinic and asking them about their shoulder as compared with before the operation and also talking with them on the telephone. Of note, and I do not think this is necessarily the same for everybody, I did speak to an elderly 
gentleman in his 70s who had a resection a few years ago and who said that his golf game was a lot better now. I am not recommending that, but it did help him.

With regard to bilateral resections go, we treated 2 patients in this manner and, as you mentioned, 1 of them died. This patient's whole manubrium ended up being necrotic, and it was removed. She was sick the whole time, and there was no way to assess her function. She was bedridden and also obese. In the other patient we tried to preserve the manubrium. Rather than taking half the manubrium midline, we took about a third on each side to leave a little bit of the manubrium island. But again, that was 1 patient of 25 who survived. Therefore, that is what we tried to do in that situation, and that person did have a single muscle flap that covered both defects.

\section{Targeted}

The Journal of Thoracic and Cardiovascular Surgery gives you two tables of contents.

The condensed table of contents tells you at a glance what topics and authors are presented each month. The expanded table of contents gives you a brief abstract of each article. You select only those articles of most interest to you for further reading. 